\title{
Assessing the need for adaptive radiotherapy in head and neck cancer patients using an automatic planning tool
}

\author{
Natália Alves ${ }^{1}$, Joana Matos Dias ${ }^{2,3}$, Humberto Rocha ${ }^{2,3}$, Tiago Ventura ${ }^{2,4}$, Josefina Mateus ${ }^{4}$, Miguel Capela \\ Leila Khouri ${ }^{5}$, Maria do Carmo Lopes ${ }^{2,4}$ \\ ${ }^{1}$ Physics Department, Faculty of Science and Technology, University of Coimbra, Coimbra, Portugal \\ ${ }^{2}$ Instituto de Engenharia de Sistemas e Computadores de Coimbra, Coimbra, Portugal \\ ${ }^{3}$ Faculty of Economics, University of Coimbra, Coimbra, Portugal \\ ${ }^{4}$ Medical Physics Department, Instituto Português de Oncologia de Coimbra Francisco Gentil, EPE, Coimbra, Portugal \\ ${ }^{5}$ Radiotherapy Department, Instituto Português de Oncologia de Coimbra Francisco Gentil, EPE, Coimbra, Portugal
}

\begin{abstract}
Background: Unbiased analysis of the impact of adaptive radiotherapy (ART) is necessary to evaluate dosimetric benefit and optimize clinics' workflows. The aim of the study was to assess the need for adaptive radiotherapy (ART) in head and neck $(\mathrm{H} \& \mathrm{~N})$ cancer patients using an automatic planning tool in a retrospective planning study.

Materials and methods: Thirty H\&N patients treated with adaptive radiotherapy were analysed. Patients had a CT scan for treatment planning and a verification CT during treatment according to the clinic's protocol. Considering these images, three plans were retrospectively generated using the iCycle tool to simulate the scenarios with and without adaptation: 1) the optimized plan based on the planning $(\mathrm{CT} ; 2$ ) the optimized plan based on the verification CT (ART-plan); 3) the plan obtained by considering treatment plan 1 re-calculated in the verification CT (non-ART plan). The dosimetric endpoints for both target volumes and OAR were compared between scenarios 2 and 3 and the SPIDERplan used to evaluate plan quality.

Results: The most significant impact of ART was found for the PTVs, which demonstrated decreased D98\% in the non-ART plan. A general increase in the dose was observed for the OAR but only the spinal cord showed a statistical significance. The SPIDERplan analysis indicated an overall loss of plan quality in the absence of ART.

Conclusion: These results confirm the advantages of ART in H\&N patients, especially for the coverage of target volumes. The usage of an automatic planning tool reduces planner-induced bias in the results, guaranteeing that the observed changes derive from the application of ART.
\end{abstract}

Key words: radiotherapy; adaptive radiotherapy; automatic planning; head and neck cancer

Rep Pract Oncol Radiother 2021;26(3):423-432

\section{Introduction}

Head and neck $(\mathrm{H} \& \mathrm{~N})$ cancer is one of the most common types of cancer worldwide, with approximately 1.5 million new cases and 0.9 million deaths in 2018 alone [1]. In current clinical practice, a vast majority of patients with locally advanced $H \& N$ cancer require radiotherapy (RT), with or without concomitant chemotherapy. In the past decades, the treatment paradigm for RT has evolved from 3D conformal RT to intensity modulated radiotherapy (IMRT), which is the current gold standard [2]. The highly conformal dose distributions produced by IMRT lead to steep dose

Address for correspondence: Natália Alves, Physics Department, Faculty of Science and Technology, University of Coimbra, Rua Larga, P-3004 516 Coimbra, Portugal, tel: +351 9163005024; e-mail: natalia.bcalves@gmail.com 
gradients surrounding the target volumes, which are extremely sensitive to positional errors and anatomic changes. This is particularly critical in $\mathrm{H} \& \mathrm{~N}$ cases, since there are several structures at risk very close, and sometimes overlapping with the target volumes [3].

In current practice, RT treatment plans are designed individually for each patient based on a planning computed tomography ( $\mathrm{p}-\mathrm{CT}$ ), on which the target volumes and the surrounding organs at risk (OAR) are contoured by a radiation oncologist (RO), usually using also other image modalities, such as MRI or PET, and auto-segmentation tools which are already available. In the course of RT treatments (typically consisting of 5 fractions per week over 5-7 weeks), several factors can lead to anatomic changes of both the target volumes and OAR leading to deviations between the actual anatomy of the patient and the one represented on the $\mathrm{p}$-CT. These factors include daily setup variations, primary tumour or nodal volume regression or progression, alteration in muscle mass and/or fat distribution, fluid shift within the body and weight loss $[3,4]$. If unnoticed or unattended, these changes might lead to discrepancies in dose delivery, namely underdosage of the target volume, with loss of tumour control, and/or to overdosage of the normal structures, potentially producing unexpected side effects $[5,6]$. In order to correct for day-to-day positioning errors relative to the $\mathrm{p}-\mathrm{CT}$, most centres perform Image Guided Radiotherapy (IGRT) where most of those mismatches are corrected through couch shifts. However, one of the main limitations of IGRT is that internal changes in size, shape or relative position of the target volumes or OARs compared with the initial p-CT cannot be corrected by rigid translational and/or rotational couch shifts [7]. A possible solution to this problem is Adaptive Radiotherapy (ART), which aims at correcting anatomical modifications by adapting the initial dose plan to the current patient status $[8,9]$. This process requires repeated imaging with sufficient quality for treatment planning, re-contouring and re-planning, and can be done either offline, between treatment fractions, or online immediately prior to a fraction while the patient is lying on the treatment couch $[9,10]$. The most frequently implemented type of ART is offline ART, where the needed ad- justments are done between treatment sessions, since online ART (during a given treatment session) requires advanced tools, such as automatic segmentation and planning, which are not commonly available for most centres. In this work, the term ART refers to offline ART.

Various studies have reported shrinkage of both primary and nodal tumours during RT treatments. A recent review by Morgan et al. [3] reported median shrinkage rates of the primary tumour ranging from $3 \%$ to $16 \%$ in the end of the second week of treatment ( 2 studies), $7 \%$ to $48 \%$ by the end of week 4 ( 2 studies) and of $6 \%$ to $66 \%$ by the end of week 7 (14 studies), with the involved nodes presenting similar results. These volumetric variations have been associated with dosimetric consequences, such as reduction of the minimum delivered dose and loss of homogeneity in the dose distribution on the target volumes [11-13]. Conversely, some studies report that, despite the existence of volume reduction, dosimetric coverage of target volumes tends to be robust to anatomical changes [14-16].

As for the organs at risk, Brouwer et al. [8] published a comprehensive review analysing 51 papers that reported anatomical and dosimetric changes in several OARs. The most highlighted organs were the parotid glands which were reported to reduce during treatment (average of $26 \% \pm 11 \%$ ) and to receive higher doses than planned, with an average increase of $2.2 \pm 2.6 \mathrm{~Gy}$ in the mean delivered dose. Furthermore, five studies found significant associations between these volume changes and complications such as increased xerostomia, reduced saliva production and increased mucositis, all of which leading to patient's decreased quality of life [8]. Three studies reported the effects of RT on the submandibular glands, showing an average volume reduction of $22 \%$ (15-32\%). Regarding the spinal cord and the brainstem, several authors reported an increase in the maximum dose or in the $\mathrm{D} 1 \%$ (the dose that is received by $1 \%$ of the volume - the near-maximum dose), being the highest reported average increase of $0.2 \%$ per fraction for the spinal cord and 0.09 Gy per fraction for the brainstem [8].

The purpose of this study is to assess the need for ART in H\&N patients by evaluating the dosimetric impact of performing a replan in the course of treatment both on the target volumes and on seventeen OARs, using an automatic planning tool. 


\section{Materials and methods}

\section{Sample description}

In this study, data from $30 \mathrm{H} \& \mathrm{~N}$ patients treated with helical IMRT (Tomotherapy HD by Accuray unit) at the Portuguese Institute of Oncology Francisco Gentil in Coimbra, Portugal, between 2016 and 2019, were retrospectively analysed. The data concerned 26 patients that had been treated with radical/curative intent and 4 with palliative intent. All patients underwent Adaptive Radiotherapy according to the clinic's protocol (described in the following section), with (22 patients) or without (8 patients) concomitant chemotherapy. The treated head and neck sites are summarized in Table 2.

The majority ( 25 out of 30 ) of patients were prescribed 69.96 Gy to the tumour planning target volume (PTV-T), with two patients being prescribed $59.4 \mathrm{~Gy}$ and three being prescribed $50 \mathrm{~Gy}$. The prescription to the lymphatic nodes' PTVs (PTV-N) was either 54 Gy or 59.4 Gy. There were 3 patients who were not prescribed dose to the lymph nodes (palliative cases). Depending on the prescription scheme, the treatment was delivered in either 33 (26 patients), 28 (1 patient) or 20 (3 patients) fractions.
Table 1. Summary of patients' characteristics. The mean and standard deviation is shown for the age and initial weight.

\begin{tabular}{|l|c|}
\hline Characteristic & Value \\
\hline Age & $61.4 \pm 10.3$ \\
\hline Gender (M/F) & $27 / 3$ \\
\hline T stage $(1 / 2 / 3 / 4)$ & $1 / 1 / 6 / 22$ \\
\hline N stage $(0 / 1 / 2 / 3 /)$ & $4 / 1 / 20 / 5$ \\
\hline Initial weight $[\mathrm{kg}]$ & $62.0 \pm 15.1$ \\
\hline
\end{tabular}

All patients were properly immobilized by a thermoplastic mask, and had a planning CT scan in treatment position acquired at a median of 14.5 days before the start of treatment (range 6-20 days), using the Somatom Sensation Open scanner from Siemens, with a $3 \mathrm{~mm}$ slice thickness. The target volumes were then manually delineated by the radiation oncologist on this CT scan with the help of a contrast-enhanced diagnostic CT, whenever available, which was co-registered with the planning CT. A $5 \mathrm{~mm}$ margin between the GTV and CTV and a $3 \mathrm{~mm}$ margin between the CTV and the PTV were considered, though the margins could have been subjected to adaptations for each

Table 2. Summary of patients' target volumes in the planning (pCT) and verification $C T$ ( $v C T$ ) by treatment site and treatment intent (radical and palliative). The mean \pm standard deviation of the tumoral (PTV-T) and nodal (PTV-N1 homolateral, PTV-N2 contralateral and PTV-N bilateral) volumes are presented for each site

\begin{tabular}{|c|c|c|c|c|c|c|c|c|c|}
\hline \multirow{2}{*}{$\begin{array}{l}\text { Site } \\
\text { Radical patient }\end{array}$} & \multirow[t]{2}{*}{$\begin{array}{c}\mathrm{N}^{\circ} \\
\text { patients }\end{array}$} & \multicolumn{2}{|c|}{ PTV-T } & \multicolumn{2}{|c|}{ PTV-N1 } & \multicolumn{2}{|c|}{ PTV-N2 } & \multicolumn{2}{|c|}{ PTV-N } \\
\hline & & pCT & vCT & pCT & $\mathrm{vCT}$ & pCT & vCT & pCT & vCT \\
\hline Tongue & 4 & $\begin{array}{c}162.06 \pm \\
59.89\end{array}$ & $\begin{array}{c}214.12 \pm \\
92.47\end{array}$ & $\begin{array}{c}211.95 \pm \\
64.37\end{array}$ & $\begin{array}{c}243.65 \pm \\
91.33\end{array}$ & $\begin{array}{c}166.03 \pm \\
20.99\end{array}$ & $\begin{array}{c}175.09 \pm \\
31.52\end{array}$ & - & - \\
\hline Mouth & 1 & 567.60 & 248.78 & - & - & - & - & 231.85 & 223.08 \\
\hline Oropharynx & 7 & $\begin{array}{c}194.31 \pm \\
108.10\end{array}$ & $\begin{array}{c}181.61 \pm \\
99.88\end{array}$ & $\begin{array}{c}282.84 \pm \\
130.82\end{array}$ & $\begin{array}{c}252.71 \pm \\
103.74\end{array}$ & $\begin{array}{c}147.03 \pm \\
97.50\end{array}$ & $\begin{array}{c}146.37 \pm \\
80.97\end{array}$ & $\begin{array}{c}594.28 \pm \\
264.65\end{array}$ & $\begin{array}{c}584.01 \pm \\
219.19\end{array}$ \\
\hline Nasopharynx & 1 & 234.34 & 181.934 & 273.94 & 229.13 & 230.31 & 209.63 & & \\
\hline Hypopharynx & 8 & $\begin{array}{c}320.69 \pm \\
120.32\end{array}$ & $\begin{array}{c}410.92 \pm \\
180.53\end{array}$ & $\begin{array}{c}398.86 \pm \\
227.13\end{array}$ & $\begin{array}{c}501.53 \pm \\
317.75\end{array}$ & $\begin{array}{c}152.33 \pm \\
44.19\end{array}$ & $\begin{array}{c}156.17 \pm \\
55.02\end{array}$ & $\begin{array}{c}651.19 \pm \\
136.09\end{array}$ & $\begin{array}{c}790.61 \pm \\
265.28\end{array}$ \\
\hline Oesophagus & 1 & 9.77 & 15.57 & - & - & - & - & 142.69 & 141.51 \\
\hline Larynx & 2 & $\begin{array}{c}79.17 \pm \\
22.31\end{array}$ & $\begin{array}{c}69.53 \pm \\
11.06\end{array}$ & 272.31 & 224.55 & 137.57 & 104.00 & 368.31 & 367.63 \\
\hline Not Specified & 2 & $\begin{array}{c}115.07 \pm \\
43.04\end{array}$ & $\begin{array}{c}99.83 \pm \\
22.58\end{array}$ & $\begin{array}{c}301.15 \pm \\
67.70\end{array}$ & $\begin{array}{c}302.11 \pm \\
63.30\end{array}$ & $\begin{array}{c}125.23 \pm \\
10.32\end{array}$ & $\begin{array}{c}129.33 \pm \\
1.52\end{array}$ & - & - \\
\hline \multicolumn{2}{|c|}{ Palliative patients } & $\mathrm{pCT}$ & $\mathrm{vCT}$ & pCT & vCT & pCT & vCT & pCT & $\mathrm{vCT}$ \\
\hline Oropharynx & 1 & 152.85 & 159.69 & - & - & - & - & - & - \\
\hline Mouth & 1 & 64.41 & 102.94 & - & - & - & - & - & - \\
\hline Nasal Cavity & 2 & $\begin{array}{c}243.10 \pm \\
65.81\end{array}$ & $\begin{array}{c}271.17 \pm \\
21.98\end{array}$ & - & - & - & - & - & - \\
\hline
\end{tabular}


specific clinical case. The OARs were delineated with the aid of an atlas-based auto segmentation system (ABAS V 2.0) which was locally configured, although verification and (if needed) correction of the structures by the radiation oncologist was always performed. The 17 structures considered in this study were the spinal cord, brainstem, parotid glands, thyroid, submandibular glands, mandible, constrictor muscle, oral cavity, lens, retinas, optic nerves, ears, chiasm, larynx, oesophagus, lungs and pituitary gland.

\section{Adaptive radiotherapy protocol}

In the clinic's routine practice, patients treated with Helical Tomotherapy undergo a daily megavoltage CT (MV-CT) scan, before each treatment fraction, to identify the differences between the current treatment position and the one established in the $\mathrm{p}$-CT. The MV-CT is the CTrue scan mode in tomotherapy which uses the $6 \mathrm{MV}$ beam tuned to 3.5 MV and the acquisition pitch corresponds to 3 $\mathrm{mm}$ thickness reconstructed images.

A rigid registration is then performed between the MV-CT and the p-CT, and a transformation vector calculated, which is translated into rigid couch and roll angle shifts to correct for positional errors.

If significant discrepancies between the images are observed after the couch corrections, the medical physicist is alerted, and a dose calculation is performed on the daily scan using the Planned Adaptive software module version 5.1.0.6 from Accuray. This dose distribution is then revised by the responsible radiation oncologist who determines if a new treatment plan is needed. Usually, a difference over $3 \%$ in the PTV minimum dose leads to re-planning. Also, the risk of overdosage in critical structures, such as the spinal cord, would lead to re-planning; however, the initial treatment plan has usually spinal doses well below tolerance.

If a replan is required, a second CT (verification $\mathrm{CT}$ ) is acquired following the same protocol as the planning CT which consists of $3 \mathrm{~mm}$ slice thickness on a dedicated big-bore CT simulator (Siemens Sensation Open). Both the target volumes and OAR are propagated from the $\mathrm{p}$-CT to the verification CT by rigid and deformable image registration using Velocity AI, version 3.2. These volumes are then manually corrected by the radiation oncologist and a new plan is calculated.
All patients in the study were treated using Adaptive Radiotherapy, following the clinic's established protocol described above. The verification CT was acquired at an average of 18 days (median $=14$ days, standard deviation $=10$ days) after the start of treatment. The volumes of the targets (both tumoral and nodal) are shown in Table 2 for the planning and verification CT by treatment site and treatment intent.

\section{Automatic plans generation}

For each patient in the cohort, three new dose distributions were generated for the exclusive purpose of the present study:

- the dose distribution generated by the automatic planning tool iCycle17, based on the planning CT. This plan represents the original treatment plan;

- the dose distribution generated by the automatic planning tool iCycle17, based on the verification CT. This plan was generated independently from the original plan and represents the scenario where adaptation occurs (ART-plan);

- the dose distribution obtained by applying the fluence and beam arrangement resulting from the original plan in 1), but now calculated on the verification CT. This plan represents the hypothetical scenario where no adaptation occurs, and the original plan would be delivered until the end of treatment despite the anatomical modifications in the patient (non-ART plan).

In iCycle [17], plan generation is guided by a user defined wish-list, which contains both clinical constraints that must be strictly met, and prioritized objectives to be optimized. The optimization occurs through a constraint-based method, which generates a single Pareto optimal IMRT solution for a given set of beams. The wish-list used for the generation of the original and ART plans (scenarios 1 and 2) were built according to the guidelines established by Ventura et al. [18].

\section{Dosimetric analysis}

The dosimetric endpoints for both target volumes and OARs were compared between the adaptive and the non-adaptive plan, to assess the impact of ART. For the target volumes the dose received by $98 \%$ of the volume (D98\%) was considered and, for the OARs, the maximum and mean doses were considered for organs with serial and parallel architectures, respectively. 
The normality and homogeneity of the variance were assessed using the Shapiro-Wilk normality test and Levene's test, respectively, in order to determine whether parametric or non-parametric tests were suitable. Then, since all variables were non-parametric, the Wilcoxon sign ranked test, with a significance level of 0.05 , was used to assess statistical significance.

\section{Quality assessment and comparison of plans}

The dose distributions obtained for the ART-plan and the non-ART plan were assessed and compared using the SPIDERplan quality assessment tool. This tool, described in detail by Ventura et al. [19] scores each structure of interest using a score function based on targets/OAR clinical constraints. These scores are inversely proportional to the quality of the plan regarding that structure, meaning that the closer the score is to zero the better the sparing of the OAR or coverage of the target volume is. A score lower than unity is achieved when all planning aims are met, and a score higher than 1 means that the plan does not comply with the required dose constraints. The structures are organized within groups and weights, which reflect the radiation oncologist's clinical preferences, are assigned to both groups and structures. The scores associated with each structure are then combined so that group scores and a global plan score reflecting the clinical preferences are calculated. The results are then presented in an intuitive graphical representation through customized radar plots.

In this study, the structures were divided into six groups (PTV group, Critical group, DigestOral group, Bone group, Optics group and Other group) which were assigned different weights $(50 \%, 30 \%$, $5 \%, 3.5 \%, 10 \%$ and $1.5 \%$, respectively) [18]. The different group structures are established based on institutional or international protocols, depending on factors like the pathology and clinical preferences, and the different weights were assigned according to the group clinical relevance [19].

All automatic plans were retrospectively generated using the imaging data available from the treated patients, with no extra images being acquired for the purpose of this work. Furthermore, all clinical and imaging data were completely anonymized prior to the inclusion in the study.

\section{Results}

The results of the dosimetric and the SPIDERplan quality analysis for every target volume and each OAR are shown in Table 3. The structures are organized in the table by the respective SPIDERplan groups, starting with the PTV group, and moving sequentially to the Critical, DigestOral, Bone, Optics and Other groups.

Figure 1 shows the SPIDERplan plot for a representative patient. The red line represents the structures' scores for the ART plan and the yellow line, the scores for the non-ART plan. It is clear that the scores for the ART plan are generally closer to the centre of the plot, meaning that they comply better with the aimed/tolerance value. The largest differences are observed for the target volumes (PTV group), and for the DigestOral group. Furthermore, for this specific patient, a significant improvement is achieved in the spinal cord with the ART plan clearly complying with the dose constraint for this OAR (score below 1), which is not the case for the non-ART plan (score above the radius 1 circle in the diagram). In fact, the scores in the ART plan are all bellow 1 (inner circle) with the exception of the thyroid, while the majority of the structures in the non-ART plan present a score above 1 , meaning that the required clinical constraints for plan acceptance are not achieved in this scenario.

\section{Discussion}

In this work, thirty $\mathrm{H} \& \mathrm{~N}$ cancer patients clinically treated with adaptive radiotherapy were retrospectively analysed in order to determine the dosimetric consequences that would have occurred in the absence of adaptation.

The dosimetric analysis was performed for all target volumes as well as seventeen OARs, providing a holistic view of the dosimetric consequences of ART for the quality of the planned treatment.

Looking at the dosimetric differences reported in Table 3, the results show that the most significant dosimetric impact of ART is observed in the target volumes, all of which presented much lower D98\% (or near minimum doses) in the non-ART scenario. The differences are so important that the requirement of the coverage of $95 \%$ of the PTV by $95 \%$ of the prescribed dose is not met on average by any target, leading to the conclusion that, in the 
Table 3. Results of the dosimetric and SPIDERplan analysis of the ART and non-ART plans. The PTV-T, PTV-N1, PTV-N2 and PTV-N correspond to the tumoral, nodal homolateral, nodal contralateral and nodal bilateral PTVs, respectively. For each target the correspondent prescription dose is shown in the structure column, and for every structure the reference dose according to the parameter in question (D98\%/Dmax/Dmean) is presented. The dosimetric analysis regarding the specified parameter is followed by the group score for the respective group. The mean global plan scores are also presented. p-values lower than 0.05 , representing statistical significance, are in bold and marked with an asterisk

\begin{tabular}{|c|c|c|c|c|c|c|c|}
\hline \multirow{2}{*}{ Parameter } & \multirow{2}{*}{ Structure } & \multirow{2}{*}{$\begin{array}{l}\text { Reference } \\
\text { Dose [Gy] }\end{array}$} & \multicolumn{2}{|c|}{ ART } & \multicolumn{2}{|c|}{ Non-ART } & \multirow{2}{*}{ p-value } \\
\hline & & & Mean & std & Mean & Std & \\
\hline \multirow{7}{*}{ D98\% [Gy] } & PTV-T (69 Gy) & 66.46 & 67.30 & 0.73 & 58.00 & 8.32 & $0.000^{*}$ \\
\hline & PTV-T (59.4 Gy) & 56.43 & 56.93 & 0.49 & 54.83 & 1.38 & $* *$ \\
\hline & PTV-T (50 Gy) & 47.50 & 48.80 & 1.18 & 37.2 & 6.56 & $* *$ \\
\hline & PTV-N1 (59.4 Gy) & 56.43 & 58.31 & 1.05 & 44.46 & 13.20 & $0.000^{*}$ \\
\hline & PTV-N2 (54 Gy) & 51.30 & 52.88 & 0.39 & 40.60 & 10.39 & $0.026^{*}$ \\
\hline & PTV-N2 (59.4 Gy) & 56.43 & 57.34 & 0.68 & 49.73 & 7.69 & $0.002^{*}$ \\
\hline & PTV-N (59 Gy) & 56.43 & 58.52 & 1.19 & 32.83 & 18.27 & $0.000^{*}$ \\
\hline Group Score & PTV group & & 0.98 & 0.01 & 2.45 & 5.98 & $0.000^{*}$ \\
\hline \multirow{2}{*}{ Dmax [Gy] } & Spinal Cord & 45 & 35.58 & 10.57 & 41.52 & 13.45 & $0.017^{*}$ \\
\hline & Brainstem & 54 & 15.36 & 13.74 & 19.19 & 17.07 & 0.474 \\
\hline Group Score & Critical group & & 0.54 & 0.20 & 0.65 & 0.25 & 0.070 \\
\hline \multirow{9}{*}{ Dmean [Gy] } & Left Parotid & 26 & 23.46 & 8.99 & 26.41 & 11.97 & 0.512 \\
\hline & Right Parotid & 26 & 24.00 & 10.07 & 25.11 & 11.88 & 0.522 \\
\hline & Oral Cavity & 45 & 29.36 & 9.67 & 28.45 & 9.84 & 0.695 \\
\hline & Oesophagus & 40 & 24.81 & 10.39 & 24.18 & 12.21 & 0.821 \\
\hline & Larynx & 45 & 33.33 & 8.56 & 31.64 & 11.76 & 0.678 \\
\hline & Right Submandibular Gland & 45 & 41.47 & 9.62 & 44.67 & 12.11 & 0.223 \\
\hline & Left Submandibular Gland & 45 & 41.31 & 15.39 & 41.32 & 17.39 & 0.709 \\
\hline & Lips & 25 & 17.54 & 5.83 & 17.77 & 5.64 & 0.915 \\
\hline & Constrictor Muscle & 45 & 40.83 & 12.12 & 40.71 & 13.78 & 0.726 \\
\hline Group Score & DigestOral group & & 0.79 & 0.28 & 0.82 & 0.30 & 0.773 \\
\hline Dmax [Gy] & Mandible & 66 & 63.11 & 13.55 & 65.75 & 14.41 & 0.069 \\
\hline \multirow{4}{*}{ Dmean [Gy] } & Right Ear & 45 & 6.58 & 9.20 & 9.62 & 12.85 & 0.959 \\
\hline & Left Ear & 45 & 6.10 & 9.43 & 7.48 & 11.91 & 0.959 \\
\hline & Right Cochlea & 45 & 16.82 & 18.97 & 16.09 & 20.88 & 0.559 \\
\hline & Left Cochlea & 45 & 10.38 & 17.87 & 12.13 & 22.37 & 0.515 \\
\hline Group Score & Bone group & & 0.58 & 0.35 & 0.61 & 0.36 & 0.751 \\
\hline \multirow{7}{*}{ Dmean [Gy] } & Right Lens & 12 & 1.06 & 2.86 & 1.57 & 2.99 & 0.655 \\
\hline & Left Lens & 12 & 1.50 & 3.75 & 1.46 & 2.82 & 1.000 \\
\hline & Right Optic Nerve & 50 & 4.76 & 11.61 & 6.92 & 15.01 & 0.655 \\
\hline & Left Optic Nerve & 50 & 6.57 & 14.66 & 8.90 & 17.24 & 0.634 \\
\hline & Right Retina & 45 & 1.66 & 4.35 & 2.50 & 5.13 & 0.490 \\
\hline & Left Retina & 45 & 2.06 & 5.09 & 2.50 & 5.01 & 0.743 \\
\hline & Chiasm & 50 & 2.73 & 6.10 & 5.54 & 12.37 & 0.884 \\
\hline Group Score & Optics group & & 0.18 & 0.45 & 0.24 & 0.49 & 0.490 \\
\hline \multirow{4}{*}{ Dmean [Gy] } & Right Lung & 5 & 2.34 & 1.70 & 2.74 & 3.08 & 0.848 \\
\hline & Left Lung & 5 & 2.26 & 1.69 & 2.53 & 2.87 & 0.763 \\
\hline & Thyroid & 27.5 & 35.81 & 12.65 & 37.03 & 13.18 & 0.750 \\
\hline & Pituitary & 60 & 3.03 & 10.01 & 3.97 & 10.85 & 0.961 \\
\hline Group Score & Other group & & 0.71 & 0.35 & 0.78 & 0.51 & 0.554 \\
\hline Global score & Plan score & & 0.77 & 0.09 & 1.62 & 3.34 & $0.000^{*}$ \\
\hline
\end{tabular}

**For the tumoral volumes prescribed 59.4 and 50 Gy there were not enough patients in each group for a statistical analysis to be meaningful ( 2 and 3 patients, respectively) 


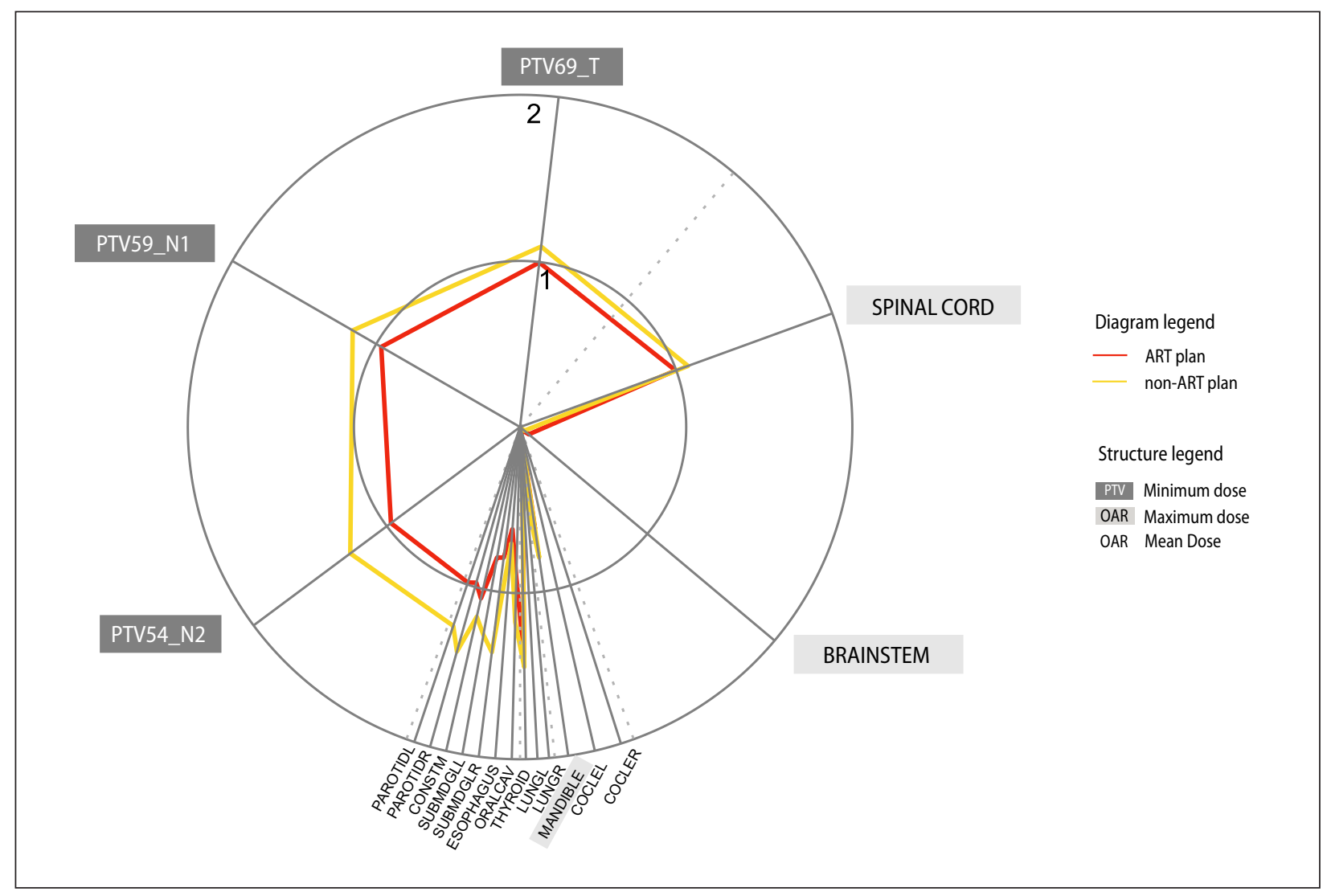

Figure 1. SPIDER plan radar plot for one representative patient. The red line represents the scores for the ART-plan for each structure and target volume while the yellow line shows the scores for the non-ART plan. The angular amplitude assigned to each structure reflects the magnitude of the weight attributed to the group to which the structure belongs. The dotted grey lines represent the separation of the different structure groups

absence of a replan, dose coverage and tumoral control would be significantly impaired.

In fact, under the non-ART scenario, only three patients met the tumour PTV clinical constraint of $98 \%$ of the volume receiving at least $95 \%$ of the prescribed dose. Regarding the nodal PTVs, there were also three patients who met this constraint, but no patient achieved this result for all their targets. In other words, in the absence of ART, there was always at least one target which did not meet the clinical objective for dose coverage. These results are in line with previously published works that stablish a link between reduced tumoral coverage and the absence of replanning $[13,20]$, with one study by Castelli et al. (2018) [21] reporting tumour underdosage in $76 \%$ of the 37 oropharyngeal patients included in their analysis during IMRT without adaptation.

These significant loss in target coverage may be associated with the anatomical modification of the target volumes, which has been reported to be common in $\mathrm{H} \& \mathrm{~N}$ treatments 3 . As can be seen in Table 2, quite significant variations in the volume of the targets were observed between the planning and verification CTs. It is worth pointing out that these variations were not homogeneous and, while most sites presented an average reduction of the volumes, there were three sites where average growth was observed, as well as within the palliative patients. Nevertheless, this heterogeneity can be attributed to the small number of patients in each site, which is also evident in the high standard deviations of the volumes.

Furthermore, it is also clear that the standard deviation of the doses is much higher in the non-ART scenario for all structures (Tab. 3). This greatly increased variance implies that not performing ART could contribute to more unpredictable side effects. The actual dosimetric consequences are highly patient specific, reinforcing the need for an individual assessment and identification of patients for ART. These results are corroborated by the SPIDERplan 
score for the PTV group, with both mean value and standard deviation significantly higher for the non-ART scenario. This score is also the only of the six groups which is greater than one, meaning that the minimum clinical criterion is not met for the target volumes in the absence of a replan.

Regarding the OAR, most structures show an increase in the mean/maximum dose in the non-ART scenario, as can be seen in Table 3 . The majority of the published literature on the dosimetric impact of ART in OAR focuses on the parotid glands, due to their well-known radiosensitivity which is associated with impaired salivary production, xerostomia and reduced quality of life $[3,8]$. There are currently relatively few studies which focus on other OARs that can also have a significant impact on the patients' life if overly irradiated, including highly critical organs, such as the spinal cord and the brainstem $[3,8]$. Our results clearly show that, although there is an increase in the mean dose to the parotids in the absence of ART, it is not statistically significant, and the most affected OAR is the spinal cord. The maximum dose to the spinal cord increased approximately 6 Gy in average, but a very high dose variation across patients was observed. This is in line with the results from other studies which report excess dose to the spinal cord, with Hansen et al. [22] reporting an increase in the maximum dose ranging from 0.2 to $15.5 \mathrm{~Gy}$ in all patients of a 13-patient cohort, and Chitapanarux et al. [23] reporting a decrease in the Dmax to the spinal cord with ART in $95 \%$ of the patients in their cohort, with a dose difference ranging from 1.6 to 5.9 Gy. The average maximum dose to the spinal cord is still below the clinical threshold of $45 \mathrm{~Gy}$ in the ART plans, even with the observed increase. However, it is reasonable to infer that this value could escalate above the limit in the upcoming weeks of treatment, if the increase is not detected, or be a drawback in case of a future tumour recurrence needing re-irradiation.

There were no observed differences on the dosimetric impact of ART between the group which was prescribed $69.96 \mathrm{~Gy}$ (25 patients) and the remaining patients ( 5 patients), meaning that the dosimetric pattern when comparing ART and non-ART scenarios is not dependent on the prescribed dose, at least for the reduced patient sample included in our study.
Despite the most significant discrepancies emerging for the target volumes and the spinal cord, the SPIDERplan global score, which gives a measure of the overall plan quality by performing a weighted score of the six different groups of structures, is also significantly higher for the non-ART scenario, with a much higher standard deviation compared to the ART case. This shows that the individual differences observed for each structure correspond, in fact, to an overall loss of plan quality, which could potentially lead to a lower treatment outcome.

Radiotherapy treatment planning is still considered a combination of science and art, being highly dependent on planners' experience and skills [24]. Due to the intrinsic complexity of the optimization task planners are faced with, it has been shown that inter and intra planner variability plays an important role in the quality of the output plans [25]. This variability can introduce a bias in the studies assessing the impact of adaptive replanning, since not only the same planner can produce different quality plans at different time points, but also the adaptive plan may not be designed by the original medical physicist due to the clinic's workload. Furthermore, depending on the nature and degree of the anatomical modification leading to the need for adaptive planning, the second plan can be of a significantly higher or lower complexity than the first one.

Additionally, when the second plan is built, the planner has already information regarding the original plan and, therefore, actively attempts to improve the dose distributions that were previously achieved. This can also introduce an improvement in the adaptive plan's dose distribution that is not necessarily due to the necessity of adaptation arising from anatomic variations, skewing the dosimetric analysis in the direction of a benefit from ART that may not be realistic.

All of these factors make it difficult to determine to which extent the observed dosimetric differences between the application or not of ART that have been found in the literature are in fact due to the adaptation of the plan, as opposed to the previously mentioned aspects.

To the best of our knowledge, this is the first study to use an automatic planning tool to generate the dose distributions corresponding to the adaptive and non-adaptive scenarios, thus eliminating other sources of differences between the 
plans and allowing for a more unbiased analysis of the dosimetric impact of ART both in targets and OAR. It is important to note that the iCycle tool is a powerful optimizer that inherently produces high quality dose distributions, which could be the reason why no statistically significant differences were found in most OARs. Although these results highlight the advantages of ART in head and neck patients, especially regarding the preservation of target coverage, the presented methodology has some limitations. The dose distributions are evaluated in a single time point of treatment without considering the total delivered dose and the number of patients is relatively small. Nevertheless, the results underline the necessity to include close monitoring of the target volume's coverage in current clinical practice, to prevent severe target underdosage. Further research on the long-term impact of ART in patient survival and disease progression is still necessary to fully understand the impact and necessity of incorporating ART into routine clinical practice.

\section{Conclusion}

This study shows that the introduction of one adaptive replan during treatment translates into statistically significant differences in the coverage of the target volumes as well as the dose to OAR. Of the seventeen analysed OARs, most showed increased doses without ART, with the spinal cord presenting the only statistically significant differences. Overall plan quality was also impaired without ART, as is shown by a significant increase in the global plan score obtained using the SPIDERplan assessment tool when compared with ART.

The usage of an automatic planning tool eliminates any bias arising from inter/intra planner variability, guaranteeing that the observed dosimetric differences in fact arise from the application or not of ART.

\section{Conflict of interests}

None declared.

\section{Funding}

This study has been funded by national funds, through FCT, Portuguese Science Foundation, under projects POCI-01-0145-FEDER-028030 and UIDB/00308/2020.

\section{Acknowledgments}

The authors would like to express their gratitude to Sebastiaan Breedveld and Ben Heijmen for making available the multicriterial optimization of IMRT plans system iCycle.

\section{References}

1. Bray F, Ferlay J, Soerjomataram I, et al. Global cancer statistics 2018: GLOBOCAN estimates of incidence and mortality worldwide for 36 cancers in 185 countries. CA Cancer J Clin. 2018; 68(6): 394-424, doi: 10.3322/ caac.21492, indexed in Pubmed: 30207593.

2. Chin D, Boyle GM, Porceddu S, et al. Head and neck cancer: past, present and future. Expert Rev Anticancer Ther. 2006; 6(7): 1111-1118, doi: 10.1586/14737140.6.7.1111, indexed in Pubmed: 16831082.

3. Morgan HE, Sher DJ. Adaptive radiotherapy for head and neck cancer. Cancers Head Neck. 2020; 5: 1, doi: 10.1186/ s41199-019-0046-z, indexed in Pubmed: 31938572.

4. Castadot $P$, Lee JA, Geets $X$, et al. Adaptive radiotherapy of head and neck cancer. Semin Radiat Oncol. 2010; 20(2): 84-93, doi: 10.1016/j.semradonc.2009.11.002, indexed in Pubmed: 20219546.

5. Barker JL, Garden AS, Ang KK, et al. Quantification of volumetric and geometric changes occurring during fractionated radiotherapy for head-and-neck cancer using an integrated CT/linear accelerator system. Int J Radiat Oncol Biol Phys. 2004; 59(4): 960-970, doi: 10.1016/j. ijrobp.2003.12.024, indexed in Pubmed: 15234029.

6. Yousuf A, Qureshi B, Hussain A, et al. SU-E-J-81: Adaptive Radiotherapy for IMRT Head \& Neck Patient in AKUH. Med Phys. 2015; 42(6 Part 8): 3282-3282, doi: 10.1118/1.4924168.

7. Yan Di, Lockman D, Martinez A, et al. Computed tomography guided management of interfractional patient variation. Semin Radiat Oncol. 2005; 15(3): 168-179, doi: $10.1016 /$ j.semradonc.2005.01.007, indexed in Pubmed: 15983942.

8. Brouwer CL, Steenbakkers RJ, Langendijk JA, et al. Identifying patients who may benefit from adaptive radiotherapy: Does the literature on anatomic and dosimetric changes in head and neck organs at risk during radiotherapy provide information to help? Radiother Oncol. 2015; 115(3): 285-294, doi: 10.1016/j.radonc.2015.05.018, indexed in Pubmed: 26094076.

9. Böck M. On adaptation cost and tractability in robust adaptive radiation therapy optimization. Med Phys. 2020; 47(7): 2791-2804, doi: $10.1002 / \mathrm{mp} .14167$, indexed in Pubmed: 32275778.

10. Green OL, Henke LE, Hugo GD. Practical Clinical Workflows for Online and Offline Adaptive Radiation Therapy. Semin Radiat Oncol. 2019; 29(3): 219-227, doi: 10.1016/j. semradonc.2019.02.004, indexed in Pubmed: 31027639.

11. Bhide SA, Davies M, Burke $K$, et al. Weekly volume and dosimetric changes during chemoradiotherapy with intensity-modulated radiation therapy for head and neck cancer: a prospective observational study. Int J Radiat Oncol Biol Phys. 2010; 76(5): 1360-1368, doi: 10.1016/j. ijrobp.2009.04.005, indexed in Pubmed: 20338474. 
12. Mnejja W, Daoud H, Fourati N, et al. Dosimetric impact on changes in target volumes during intensity-modulated radiotherapy for nasopharyngeal carcinoma. Rep Pract Oncol Radiother. 2020; 25(1): 41-45, doi: 10.1016/j. rpor.2019.12.012, indexed in Pubmed: 31889919.

13. Ahn PH, Chen CC, Ahn Al, et al. Adaptive planning in intensity-modulated radiation therapy for head and neck cancers: single-institution experience and clinical implications. Int J Radiat Oncol Biol Phys. 2011; 80(3): 677-685, doi: 10.1016/j.ijrobp.2010.03.014, indexed in Pubmed: 20619553.

14. Castadot $P$, Geets $X$, Lee JA, et al. Adaptive functional image-guided IMRT in pharyngo-laryngeal squamous cell carcinoma: is the gain in dose distribution worth the effort? Radiother Oncol. 2011; 101(3): 343-350, doi: 10.1016/j.radonc.2011.06.011, indexed in Pubmed: 21724283.

15. O'Daniel JC, Garden AS, Schwartz DL, et al. Parotid gland dose in intensity-modulated radiotherapy for head and neck cancer: is what you plan what you get? Int J Radiat Oncol Biol Phys. 2007; 69(4): 1290-1296, doi: 10.1016/j. ijrobp.2007.07.2345, indexed in Pubmed: 17967319.

16. Wu Q, Chi Y, Chen PY, et al. Adaptive replanning strategies accounting for shrinkage in head and neck IMRT. Int J Radiat Oncol Biol Phys. 2009; 75(3): 924-932, doi: 10.1016/j. ijrobp.2009.04.047, indexed in Pubmed: 19801104.

17. Breedveld S, Storchi PRM, Voet PWJ, et al. iCycle: Integrated, multicriterial beam angle, and profile optimization for generation of coplanar and noncoplanar IMRT plans. Med Phys. 2012; 39(2): 951-963, doi: 10.1118/1.3676689, indexed in Pubmed: 22320804.

18. Ventura T, Rocha H, da Costa Ferreira B, et al. Comparison of two beam angular optimization algorithms guided by automated multicriterial IMRT. Phys Med. 2019; 64:
210-221, doi: 10.1016/j.ejmp.2019.07.012, indexed in Pubmed: 31515022.

19. Ventura T, Lopes M, Ferreira B, et al. SPIDERplan: A tool to support decision-making in radiation therapy treatment plan assessment. Rep Pract Oncol Radiother. 2016; 21(6): 508-516, doi: 10.1016/j.rpor.2016.07.002, indexed in Pubmed: 27698591.

20. Zhang P, Simon A, Rigaud B, et al. Optimal adaptive IMRT strategy to spare the parotid glands in oropharyngeal cancer. Radiother Oncol. 2016; 120(1): 41-47, doi: 10.1016/j. radonc.2016.05.028, indexed in Pubmed: 27372223.

21. Castelli J, Simon A, Rigaud B, et al. Adaptive radiotherapy in head and neck cancer is required to avoid tumor underdose. Acta Oncol. 2018; 57(9): 1267-1270, doi: 10.1080/0 284186X.2018.1468086, indexed in Pubmed: 29706107.

22. Hansen EK, Bucci MK, Quivey JM, et al. Repeat CT imaging and replanning during the course of IMRT for head-andneck cancer. Int J Radiat Oncol Biol Phys. 2006; 64(2): 355-362, doi: 10.1016/j.ijrobp.2005.07.957, indexed in Pubmed: 16256277.

23. Chitapanarux I, Chomprasert K, Nobnaop W, et al. A dosimetric comparison of two-phase adaptive intensity-modulated radiotherapy for locally advanced nasopharyngeal cancer. J Radiat Res. 2015; 56(3): 529-538, doi: 10.1093/ jrr/rru119, indexed in Pubmed: 25666189.

24. Breedveld S, Craft D, Haveren Rv, et al. Multi-criteria optimization and decision-making in radiotherapy. Eur J Operat Res. 2019; 277(1): 1-19, doi: 10.1016/j.ejor.2018.08.019.

25. Batumalai $V$, Jameson MG, Forstner DF, et al. How important is dosimetrist experience for intensity modulated radiation therapy? A comparative analysis of a head and neck case. Pract Radiat Oncol. 2013; 3(3): e99-e9e106, doi: 10.1016/j.prro.2012.06.009, indexed in Pubmed: 24674377. 